\title{
Screening of biomarkers in cervical squamous cell carcinomas via gene expression profiling
}

\author{
BING CHEN, CHUNDONG LI, LEI ZHANG, JIAHUI LV and YING TONG \\ Department of Gynaecology and Obstetrics, General Hospital of The Air Force, Beijing 100142, P.R. China
}

Received December 9, 2014; Accepted August 27, 2015

DOI: $10.3892 / \mathrm{mmr} .2015 .4322$

\begin{abstract}
In the present study, gene expression profiles of high-grade squamous intraepithelial lesions (HSIL) and invasive cervical squamous cell carcinomas (CSCC) were analyzed using bioinformatic tools to identify key genes and potential biomarkers. Analyses of differentially expressed genes (DEGs) were performed for HSIL vs. normal control and invasive CSCC vs. normal control tissues using the Limma package in $\mathrm{R}$. Pathway enrichment analysis was performed using KOBAS. A protein-protein interaction (PPI) network for the DEGs in invasive CSCC was constructed using String. Functional enrichment analysis was performed for the DEGs in the PPI network using DAVID. Relevant small molecules were predicted using Cmap. A total of 633 and 881 DEGs were identified in HSIL and invasive CSCC, respectively, and the two groups had 305 DEGs in common. Genes associated with the mitogen-activated protein kinase signaling pathway were enriched in the HSIL, while cell cycle-associated genes were over-represented in invasive CSCC. The PPI network, containing 72 upregulated genes and 434 edges, was illustrated. Functional enrichment analysis showed that the cell cycle was the most significant gene ontology term. A total of six small molecules associated with the pathology of CSCC were identified, including the anti-cancer drug piperlongumine, which showed a negative correlation. The findings of the present study not only enhanced the current understanding of the pathogenesis of CSCC, but may also be a basis for the development of novel therapies.
\end{abstract}

\section{Introduction}

Cervical cancer is the second most common cancer in women worldwide with the fifth highest mortality rate $(1,2)$. Squamous cell carcinomas are the most common type, accounting for

Correspondence to: Professor Ying Tong, Department of Gynaecology and Obstetrics, General Hospital of The Air Force, 30 Fucheng Road, Haidian, Beijing 100142, P.R. China

E-mail: 13911558200@139.com

Key words: biomarker, cervical squamous cell carcinoma, differentially expressed gene, high-grade squamous intraepithelial lesions, protein-protein interaction network
$80-85 \%$ of all cervical cancers (3). Infection with human papilloma virus is the greatest risk factor for cervical cancer (4), followed by smoking (5). The five-year relative survival rate for the earliest stage of invasive cervical cancer is $92 \%$; however, the prognosis is significantly lower when metastasis is present, suggesting the importance of early diagnosis.

Studies have identified pathways associated with the pathogenesis of cervical cancer, particularly the molecular mechanisms underlying its invasiveness. Wnt signaling was reported to be involved in the pathogenesis of cervical cancer (6). Tumor necrosis factor- $\alpha$ (TNF- $\alpha$ ) is a pro-inflammatory cytokine, which has been implicated in several cancers. Duarte et al (7) reported that G-308A TNF- $\alpha$ polymorphism is associated with an increased risk of invasive cervical cancer. Chan et al (8) indicated that overexpression of forkhead box M1 transcription factor is associated with cervical cancer progression and pathogenesis. Murphy et al (9) performed an immunocytochemical analysis to reveal that p16INK4A, CDC6 and MCM5 are predictive biomarkers in cervical pre-invasive neoplasia and cervical cancer. Microarray technology is also widely adopted in the discovery of crucial genes. Song et al (10) identified several candidate genes associated with invasion of cervical cancer via microarray analysis of normal cervix, in situ carcinoma and invasive cervical cancer tissues. Zhai et al (11) identified genes contributing to the invasive properties of cervical carcinoma cells. However, as these findings have not resulted in an improved outcome for patients with cervical carcinoma, additional study is required.

The present study, analyzed gene expression profiles of high-grade squamous intraepithelial lesions (HSIL) and invasive cervical squamous cell carcinomas (CSCC) with currently available bioinformatic tools, attempting to identify crucial genes in the pathogenesis of CSCC as well as potential biomarkers for diagnosis or prognosis.

\section{Materials and methods}

Microarray data. A gene expression data set [accession no. GSE7803 (11)], including 10 normal squamous cervical epitheilial, 7 HSIL and 21 invasive CSCC samples, was downloaded from Gene Expression Omnibus (http://www.ncbi.nlm. nih.gov/geo/). Gene expression levels were determined using the Affymetrix Human Genome U133A Array (no. HG-U133A; Affymetrix Inc., Santa Clara, CA, USA), and probe annotations were acquired. 
Screening of DEGs. The 22,283 probes were mapped into 20,967 genes. A $\log ^{2}$ transformation was applied on the gene expression levels (12). Analysis of differentially expressed genes (DEGs) was performed for pre-invasive cervical squamous cell carcinomas vs. normal control and invasive cervical squamous cell carcinomas vs. normal control groups using the Limma package (13) in R. Multiple testing correction according to the Benjamini-Hochberg (BH) method (14) was applied to the $\mathrm{P}$-values and the false discovery rate (FDR) was calculated. FDR $<0.05$ was set as the cut-off value to screen out significant DEGs.

To identify genes associated with the invasiveness of CSCC, DEGs in HSIL were compared with those in invasive CSCC.

Cluster analysis. Two-way cluster analysis was performed using the expression levels of the DEGs with package pheatmap in R (15). An Euclidean distance was adopted in the analysis.

Pathway enrichment analysis. Pathway enrichment analysis was performed for the DEGs using KOBAS (16). The statistical method is based on cumulative hypergeometric distribution and $\mathrm{P}<0.05$ was set as the threshold to filter out significantly over-represented biological pathways.

Construction of a protein-protein interaction (PPI) network. Proteins are involved in complex interaction networks to fulfil certain biological functions. Therefore, revealing the PPI is a useful method to identify molecular mechanisms. A PPI network was constructed for the DEGs of invasive CSCC using String (17), which was then visualized by Cytoscape (18).

Functional enrichment analysis. Functional enrichment analysis was performed for the DEGs in the PPI network using the Database for Annotation, Visualization and Integration Discovery (DAVID; http://david.abcc.ncifcrf.gov/) (19) online tool. The statistical method is based on hypergeometric distribution. FDR $<0.05$ was set as the cut-off value.

Prediction of relevant small molecules. Connectivity map (Cmap) was designed to link gene patterns associated with disease to corresponding patterns produced by drug candidates $(20,21)$. Relevant small molecules were predicted using the DEGs and those with Iscorel $>0.9$ were retained.

\section{Results}

Differentially expressed genes. Compared with normal controls, 633 and 881 DEGs were identified in HSIL and invasive CSCC, respectively.

The two groups of DEGs were compared and 305 genes were found to be common between HSIL and invasive CSCC (Fig. 1).

Cluster analysis. To verify the reliability of the DEG results, two-way cluster analysis was performed with unique DEGs of HSIL and invasive CSCC (Fig. 2). HSIL as well as invasive CSCC were clearly separated from normal controls, confirming the reliability of the DEG analysis.

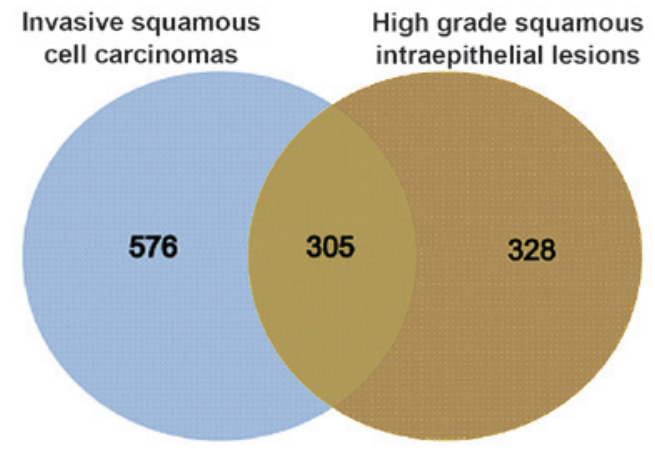

Figure 1. Venn diagram displaying the number of differentially expressed genes in high-grade squamous intraepithelial lesions and invasive squamous cell carcinomas.
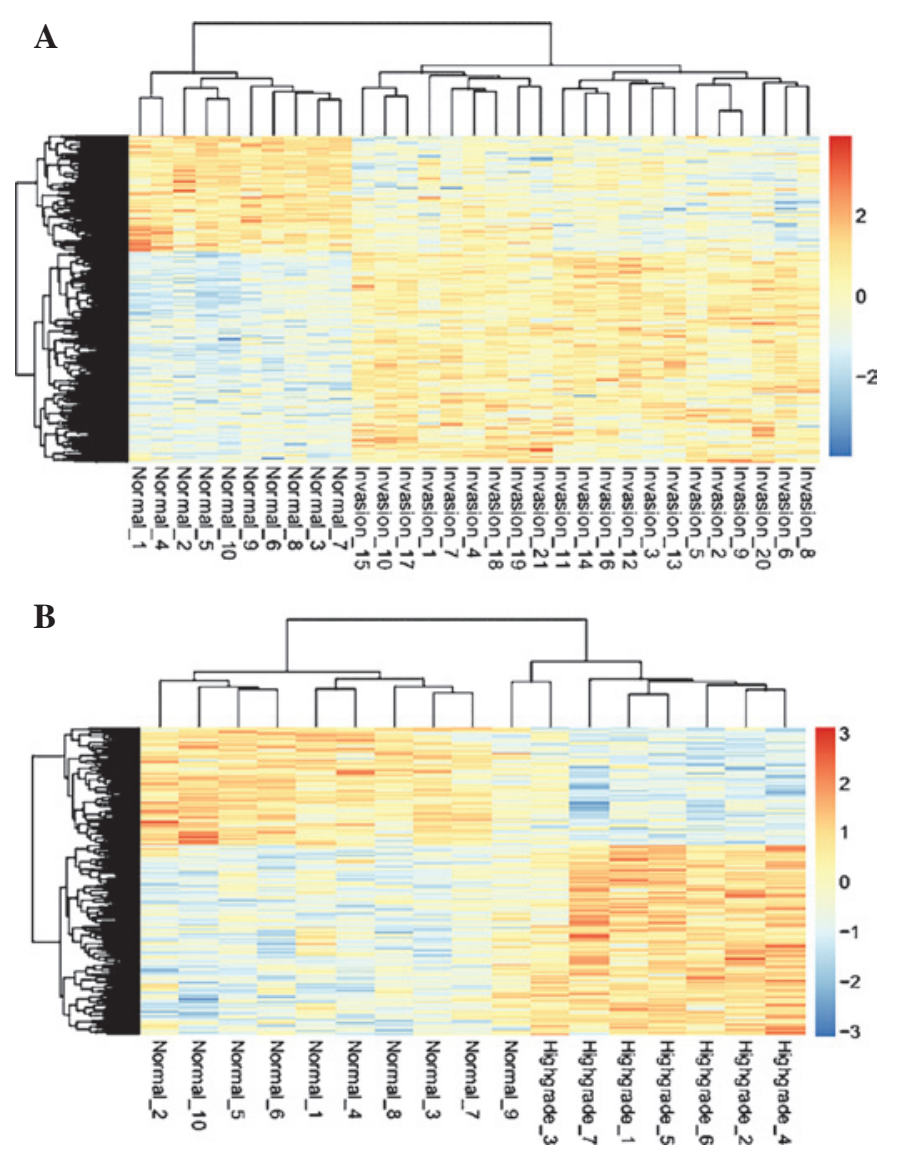

Figure 2. Hierarchical clustering of differentially expressed genes between (A) pre-invasive cervical squamous cell carcinomas and normal control as well as (B) invasive cervical squamous cell carcinomas and normal control.

Pathway enrichment analysis. Pathway enrichment analysis was performed for the unique DEGs and common DEGs of HSIL and invasive CSCC using KOBAS (Table I). the mitogen-activated protein kinase (MAPK) signaling pathway was significantly enriched in the unique DEGs of HSIL, while the cell cycle was overrepresented in the unique DEGs of invasive CSCC.

PPI network of DEGs in invasive CSCC. A PPI network was constructed for the DEGs in invasive CSCC (Fig. 3). The network consisted of 72 upregulated genes and 434 edges. 
Table I. Significantly enriched pathways in the three groups of DEGs.

A, Unique DEGs in high grade squamous intraepithelial lesions

\begin{tabular}{lll}
\hline ID & \multicolumn{1}{c}{ Pathway description } & P-value \\
\hline hsa04010 & MAPK signaling pathway & 0.004436 \\
hsa00512 & O-Glycan biosynthesis & 0.005334 \\
hsa05200 & Pathways in cancer & 0.011241 \\
hsa00531 & Glycosaminoglycan degradation & 0.013071 \\
hsa05221 & Acute myeloid leukemia & 0.049570 \\
\hline
\end{tabular}

\section{B, Common DEGs}

\begin{tabular}{llr}
\hline hsa00590 & Arachidonic acid metabolism & $8.98 \times 10^{-4}$ \\
hsa05120 & Epithelial cell signaling in Helicobacter pylori infection & 0.012220 \\
hsa00591 & Linoleic acid metabolism & 0.018788 \\
hsa03030 & DNA replication & 0.036427 \\
hsa04115 & p53 signaling pathway & 0.049630
\end{tabular}

C, Unique DEGs in invasive squamous cell carcinomas

\begin{tabular}{lll}
\hline hsa04110 & Cell cycle & $1.23 \times 10^{-10}$ \\
hsa03030 & DNA replication & $1.01 \times 10^{-5}$ \\
hsa04115 & p53 signaling pathway & $8.46 \times 10^{-5}$ \\
hsa04114 & Oocyte meiosis & $5.11 \times 10^{-4}$ \\
hsa03440 & Homologous recombination & $7.91 \times 10^{-4}$ \\
hsa05200 & Pathways in cancer & 0.003796 \\
hsa05215 & Prostate cancer & 0.010327 \\
hsa03410 & Base excision repair & 0.013273 \\
hsa03430 & Mismatch repair & 0.013275 \\
hsa04610 & Complement and coagulation cascades & 0.022293 \\
hsa03420 & Nucleotide excision repair & 0.033021 \\
\hline
\end{tabular}

DEGs, differentially expressed genes; hsa, Homo sapiens; MAPK, mitogen-activated protein kinase.

Functional enrichment analysis. Functional enrichment analysis was performed for the genes in the PPI network using the DAVID online tool. The top 10 gene ontology (GO) terms are listed in Table II. All of these terms were associated with the cell cycle, which was in accordance with the results of the pathway enrichment analysis. A total of 41 DEGs were involved in the cell cycle, including DBF4, TTK, PTTG1, CDC45, CDK1, CDC20, MCM2, MCM6, CCNB1 and MAD2L1.

Relevant small molecules. A total of six relevant small molecules were predicted by Cmap with Iscorel >0.9 (Table III). Piperlongumine was the most negatively correlated molecule. Previous studies have indicated that piperlongumine has anti-tumor activity $(22,23)$.

\section{Discussion}

In the present study, a comparative analysis of gene expression profiles was performed between HSIL, invasive CSCC and normal controls. A total of 633 and 881 DEGs were identified in HSIL and invasive CSCC, respectively. Comparison of the two groups of DEGs showed that the HSIL and CSCC groups had 305 DEGs in common. Cluster analysis results verified the confidence of the DEGs. Pathway enrichment analysis revealed that the MAPK signaling pathway was significantly enriched in the unique DEGs of HSIL, while the cell cycle was overrepresented among the unique DEGs of invasive CSCC. The MAPK pathway can be activated by diverse extracellular and intracellular stimuli and regulates a variety of cellular activities, including proliferation, differentiation, survival and death. Deregulation of MAPK pathways has been implicated in numerous human diseases, including cancer $(24,25)$. Dysregulation of the cell cycle is the most common feature of cancer $(20,26)$ and the analysis of the present study revealed that it was most significantly enriched in invasive CSCC.

To further investigate the molecular mechanisms underlying invasive CSCC, the PPI network was constructed, which included 72 upregulated genes and 434 edges. Functional enrichment analysis revealed that the cell cycle and GO terms 
Table II. Significantly enriched GO terms in the genes from the network.

\begin{tabular}{llccc}
\hline GO term & \multicolumn{1}{c}{ Function } & Count & P-value & FDR \\
\hline 0007049 & Cell cycle & 41 & $1.35 \times 10^{-33}$ & $1.96 \times 10^{-30}$ \\
0000279 & M phase & 31 & $1.37 \times 10^{-31}$ & $1.99 \times 10^{-28}$ \\
0000278 & Mitotic cell cycle & 32 & $1.43 \times 10^{-31}$ & $2.07 \times 10^{-28}$ \\
0022403 & Cell cycle phase & 33 & $1.49 \times 10^{-31}$ & $2.16 \times 10^{-28}$ \\
0007067 & Mitosis & 27 & $3.88 \times 10^{-30}$ & $5.63 \times 10^{-27}$ \\
0000280 & Nuclear division & 27 & $3.88 \times 10^{-30}$ & $5.63 \times 10^{-27}$ \\
0022402 & Cell cycle process & 35 & $4.74 \times 10^{-30}$ & $6.88 \times 10^{-27}$ \\
0000087 & M phase of mitotic cell cycle & 27 & $6.31 \times 10^{-30}$ & $9.15 \times 10^{-27}$ \\
0048285 & Organelle fission & 27 & $1.14 \times 10^{-29}$ & $1.66 \times 10^{-26}$ \\
0051301 & Cell division & 27 & $9.56 \times 10^{-27}$ & $1.39 \times 10^{-23}$ \\
\hline
\end{tabular}

FDR, false discovery rate; GO, gene ontology.

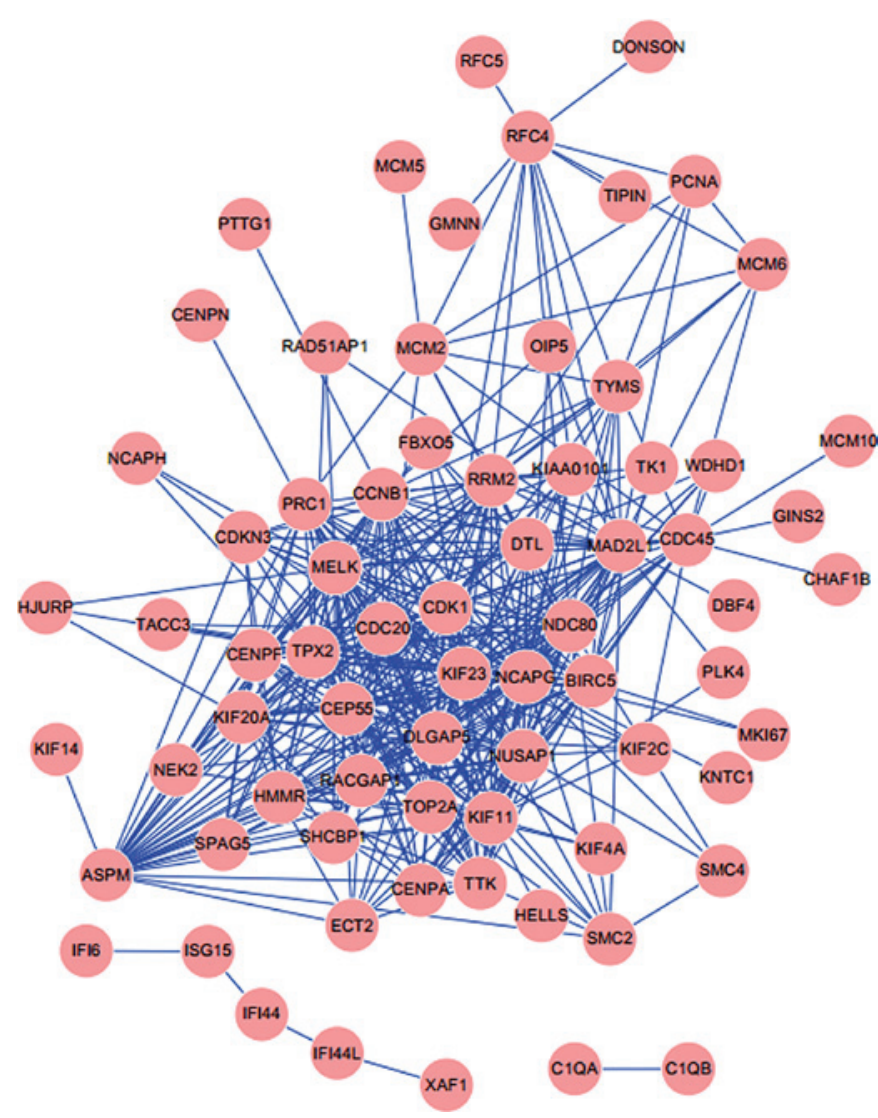

Figure 3. Protein-protein interaction network for the upregulated differentially expressed genes of invasive cervical squamous cell carcinomas.

associated with the cell cycle were enriched in the genes from the network. This finding was consistent with the results of the pathway enrichment analysis. Several of the genes identified were key genes or potential targets in invasive CSCC. NEK2 is a serine/threonine-protein kinase that is involved in mitotic regulation. Upregulation of NEK2 is observed in cell lines derived from breast cancer (27) and cervical cancer (28). Hayward and Fry (28) suggested that NEK2 contributes to chromosome instability and may be a target for chemotherapeutic intervention. DBF4 is involved in cell adhesion and
Table III. Small molecules associated with the pathology of cervical squamous cell carcinomas.

\begin{tabular}{lcr}
\hline Cmap name & Enrichment & P-value \\
\hline Piperlongumine & -0.927 & 0.01099 \\
GW-8510 & -0.915 & 0.00010 \\
Alsterpaullone & -0.911 & 0.00128 \\
Quinostatin & -0.901 & 0.01976 \\
Prestwick-692 & 0.943 & $<0.00010$ \\
Isoflupredone & 0.959 & 0.00010 \\
\hline
\end{tabular}

migration, possibly through its regulation of the arrangement of the actin cytoskeleton (29). The overexpression of DBF4 has been reported in numerous cancer types (30), and the present study revealed that it was upregulated in invasive CSCC and may contribute to the metastasis of CSCC. PTTG1 has transforming activity in vitro and tumorigenic activity in vivo, and is highly expressed in various tumor types. Depletion of PTTG1 has anti-proliferative effects in multiple tumor types (31). It also increases cell motility and promotes lymph node metastasis in esophageal squamous cell carcinoma (32). Hence, the present study speculated that PTTG1 may have a crucial role in the proliferation and mobility of cervical cancer cells. Elevated expression levels of MCM2 and MCM6 has been reported in cervical neoplasia (33), suggesting that these genes may be implicated in the development of CSCC.

Furthermore, the present study predicted associated small molecules using the expression levels of DEGs in invasive CSCC by using Cmap. Piperlongumine was the most negatively correlated molecule, which is a bioactive compound isolated from long peppers that shows selective toxicity towards a variety of cancer cell types (34). The cytotoxicity of piperlongumine has been attributed to increases in reactive oxygen species in cancer cells. Jarvius et al (35) reported that it induces inhibition of the ubiquitin-proteasome system in cancer cells. Ginzburg et al (36) further reported that piperlongumine inhibits nuclear factor- $\kappa \mathrm{B}$ activity and attenuates aggressive growth characteristics of prostate cancer cells. Piperlongumine may therefore be suitable 
for controlling invasive CSCC. This result may be useful for the development of drugs for invasive CSCC.

In conclusion, the present study identified a number of DEGs in HSIL and invasive CSCC, which may provide direction for future studies. Potential biomarkers and associated small molecules for CSCC were revealed, which may contribute to the development of novel diagnostic markers and therapeutics for CSCC.

\section{References}

1. Armstrong EP: Prophylaxis of cervical cancer and related cervical disease: A review of the cost-effectiveness of vaccination against oncogenic HPV types. J Manag Care Pharm 16: 217-230, 2010.

2. Steward BW and Wild CP: World Cancer Report. World Health Organization, Geneva, 2014.

3. Chaturvedi AK: Beyond cervical cancer: Burden of other HPV-related cancers among men and women. J Adolesc Health 46 (4 Suppl): S20-S26, 2010.

4. Schiffman M, Castle PE, Jeronimo J, Rodriguez AC and Wacholder S: Human papillomavirus and cervical cancer. Lancet 370: 890-907, 2007.

5. Gadducci A, Barsotti C, Cosio S, Domenici L and Riccardo Genazzani A: Smoking habit, immune suppression, oral contraceptive use and hormone replacement therapy use and cervical carcinogenesis: A review of the literature. Gynecol Endocrinol 27: 597-604, 2011.

6. Uren A, Fallen S, Yuan H, Usubütün A, Küçükali T, Schlegel R and Toretsky JA: Activation of the canonical wnt pathway during genital keratinocyte transformation: A model for cervical cancer progression. Cancer Res 65: 6199-6206, 2005.

7. Duarte I, Santos A, Sousa H, Catarino R, Pinto D, Matos A, Pereira D, Moutinho J, Canedo P, Machado JC and Medeiros R: G-308A TNF-alpha polymorphism is associated with an increased risk of invasive cervical cancer. Biochem Biophys Res Commun 334: 588-592, 2005.

8. Chan D, Yu S, Chiu PM, Yao KM, Liu VW, Cheung AN and Ngan HY: Over-expression of FOXM1 transcription factor is associated with cervical cancer progression and pathogenesis. J Pathol 215: 245-252, 2008.

9. Murphy N, Ring M, Heffron CC, King B, Killalea AG, Hughes C, Martin CM, McGuinness E, Sheils O and O'Leary JJ: p16INK4A, CDC6 and MCM5: Predictive biomarkers in cervical preinvasive neoplasia and cervical cancer. J Clin Pathol 58: 525-534, 2005.

10. Song JY, Lee JK, Lee NW, Jung HH, Kim SH and Lee KW: Microarray analysis of normal cervix, carcinoma in situ and invasive cervical cancer: Identification of candidate genes in pathogenesis of invasion in cervical cancer. Int J Gynecol Cancer 18: 1051-1059, 2008

11. Zhai Y, Kuick R, Nan B, Ota I, Weiss SJ, Trimble CL, Fearon ER and Cho KR: Gene expression analysis of preinvasive and invasive cervical squamous cell carcinomas identifies HOXC10 as a key mediator of invasion. Cancer Res 67: 10163-10172, 2007.

12. Fujita A, Sato JR, Rodrigues LO, Ferreira CE and Sogayar MC: Evaluating different methods of microarray data normalization. BMC bioinformatics 7: 469, 2006.

13. Smyth GK: Limma: Linear models for microarray data. In: Bioinformatics and computational biology solutions using $\mathrm{R}$ and Bioconductor Springer, pp397-pp420, 2005.

14. Benjamini $Y$ and Hochberg Y: Controlling the false discovery rate: a practical and powerful approach to multiple testing. Journal of the Royal Statistical Society. Series B (Methodological) 289-300, 1995.

15. Szekely GJ and Rizzo ML: Hierarchical clustering via joint between-within distances: Extending Ward's minimum variance method. Journal of Classification 22: 151-183, 2005

16. Xie C, Mao X, Huang J, Ding Y, Wu J, Dong S, Kong L, Gao G, Li CY and Wei L: KOBAS 2.0: A web server for annotation and identification of enriched pathways and diseases. Nucleic Acids Res 39 (Web Server Issue) W316-W322, 2011
17. Szklarczyk D, Franceschini A, Kuhn M, Simonovic M, Roth A, Minguez P, Doerks T, Stark M, Muller J, Bork P, et al: The STRING database in 2011: Functional interaction networks of proteins, globally integrated and scored. Nucleic Acids Res 39 (Database Issue) D561-D568, 2011.

18. Smoot ME, Ono K, Ruscheinski J, Wang PL and Ideker T: Cytoscape 2.8: New features for data integration and network visualization. Bioinformatics 27: 431-432, 2011.

19. Huang da W, Sherman BT and Lempicki RA: Systematic and integrative analysis of large gene lists using DAVID bioinformatics resources. Nat Protoc 4: 44-57, 2008.

20. Evan GI and Vousden KH: Proliferation, cell cycle and apoptosis in cancer. Nature 411: 342-348, 2001

21. Catania A, Urban S, Yan E, Hao C, Barron G and Allalunis-Turner J: Expression and localization of cyclin-dependent kinase 5 in apoptotic human glioma cells. Neuro Oncol 3: 89-98, 2001.

22. Kong EH, Kim YJ, Kim YJ, Cho HJ, Yu SN, Kim KY, Chang JH and Ahn SC: Piplartine induces caspase-mediated apoptosis in PC-3 human prostate cancer cells. Oncol Rep 20: 785-792, 2008.

23. Bezerra DP, Militão GC, de Castro FO, Pessoa C, de Moraes MO, Silveira ER, Lima MA, Elmiro FJ and Costa-Lotufo LV: Piplartine induces inhibition of leukemia cell proliferation triggering both apoptosis and necrosis pathways. Toxicology In Vitro 21: 1-8, 2007.

24. Kim EK and Choi EJ: Pathological roles of MAPK signaling pathways in human diseases. Biochim Biophys Acta 1802: 396-405, 2010

25. Wagner EF and Nebreda AR: Signal integration by JNK and p38 MAPK pathways in cancer development. Nat Rev Cancer 9: 537-549, 2009.

26. Kastan MB and Bartek J: Cell-cycle checkpoints and cancer. Nature 432: 316-323, 2004

27. Hayward DG, Clarke RB, Faragher AJ, Pillai MR, Hagan IM and Fry AM: The centrosomal kinase Nek2 displays elevated levels of protein expression in human breast cancer. Cancer Res 64: 7370-7376, 2004.

28. Hayward DG and Fry AM: Nek2 kinase in chromosome instability and cancer. Cancer Lett 237: 155-166, 2006.

29. Chen Y, Lu B, Yang Q, Fearns C, Yates JR III and Lee JD: Combined integrin phosphoproteomic analyses and small interfering RNA-based functional screening identify key regulators for cancer cell adhesion and migration. Cancer Res 69: 3713-3720, 2009.

30. Bonte D, Lindvall C, Liu H, Dykema K, Furge K and Weinreich M: Cdc7-Dbf4 kinase overexpression in multiple cancers and tumor cell lines is correlated with p53 inactivation. Neoplasia 10: 920-931, 2008.

31. Cho-Rok J, Yoo J, Jang YJ, Kim S, Chu IS, Yeom YI, Choi JY and Im DS: Adenovirus-mediated transfer of siRNA against PTTG1 inhibits liver cancer cell growth in vitro and in vivo. Hepatology 43: 1042-1052, 2006.

32. Ito T, Shimada Y, Kan T, David S, Cheng Y, Mori Y, Agarwal R, Paun B, Jin Z, Olaru A, et al: Pituitary tumor-transforming 1 increases cell motility and promotes lymph node metastasis in esophageal squamous cell carcinoma. Cancer Res 68: 3214-3224, 2008.

33. Malinowski DP: Multiple biomarkers in molecular oncology. I. Molecular diagnostics applications in cervical cancer detection. Expert Rev Mol Diagn 7: 117-131, 2007.

34. Liu JM, Pan F, Li L, Liu QR, Chen Y, Xiong XX, Cheng K, Yu SB, Shi Z, Yu AC and Chen XQ: Piperlongumine selectively kills glioblastoma multiforme cells via reactive oxygen species accumulation dependent JNK and p38 activation. Biochem Biophys Res Commun 437: 87-93, 2013.

35. Jarvius M, Fryknäs M, D'Arcy P, Sun C, Rickardson L, Gullbo J, Haglund C, Nygren P, Linder S and Larsson R: Piperlongumine induces inhibition of the ubiquitin-proteasome system in cancer cells. Biochem Biophys Res Commun 431: 117-123, 2013.

36. Ginzburg S, Golovine KV, Makhov PB, Uzzo RG, Kutikov A and Kolenko VM: Piperlongumine inhibits NF- $\kappa$ B activity and attenuates aggressive growth characteristics of prostate cancer cells. Prostate 74: 177-186, 2014. 